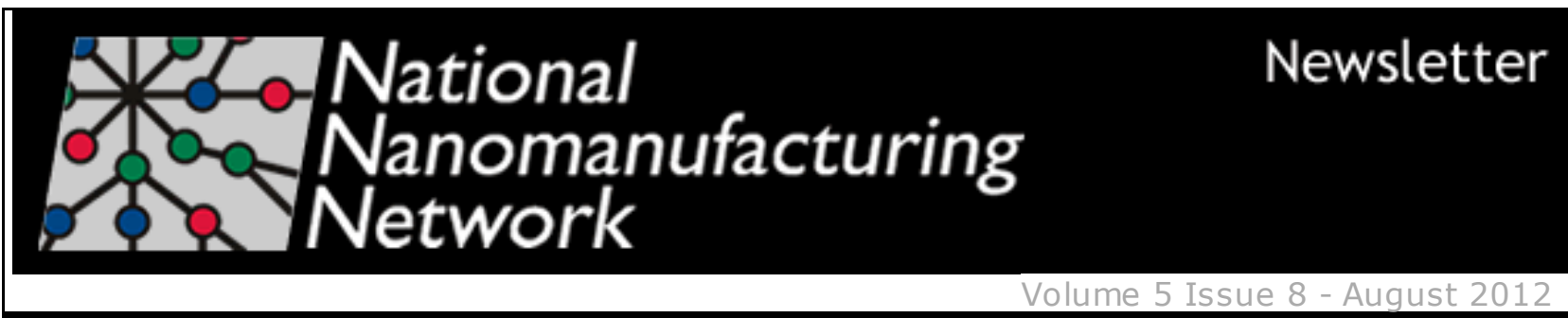

\section{Sustainable and Scalable Nanomanufacturing a Key Focus of 2012 Summit in Boston}

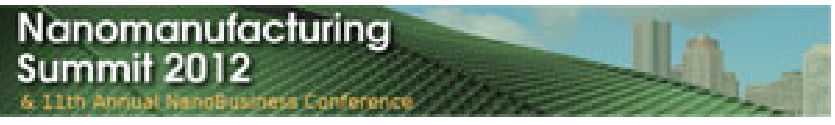

On the eve of next week's Nanomanufacturing Summit and 11th Annual NanoBusiness Conference in Boston, I'd like to take pause and reflect upon the synergistic events and interactions that have been taking place over the past several years positioning us for the nanomanufacturing revolution. While nanomanufacturing has often been a misunderstood topic or discipline within the broader topic of nanotechnology, it has gained substantial attention in recent years. Most notable has been the PCAST report on the assessment of the National Nanotechnology Initiative (NNI) in 2010, providing key recommendations for future directions in which it cited the need for increased funding and emphasis on nanomanufacturing, commercialization, and effective technology transfer. Soon thereafter, the NNI Signature Initiative on Sustainable Nanomanufacturing was announced. The goal of this interagency initiative is to establish manufacturing technologies for economical and sustainable integration of nanoscale building blocks into complex, large-scale systems. More....

Regards, Jeff Morse, Managing Director, National Nanomanufacturing Network

Learn More about the

California in the Nano Economy

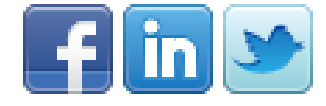

11th Annual

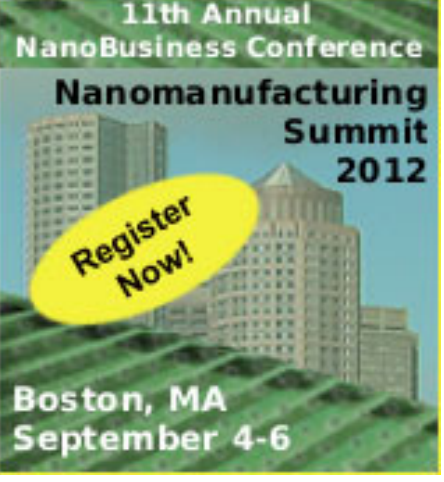

\section{Job Opportunities}

Senior Designer, Product

Development

Philips

\section{Associate Editor}

Nature Nanotechnology

Nano Device Scientist and Engineer

Aneeve Nanotechnologies

\section{Upcoming Events}

September 4-6, 2012

Nanomanufacturing Summit

September 17-22, 2012

Nanomaterials: Application \&

Properties 2012

August 20-23, 2012

IEEE Nano 2012: 12th

International Conference on Nanotechnology

View Full Calendar 


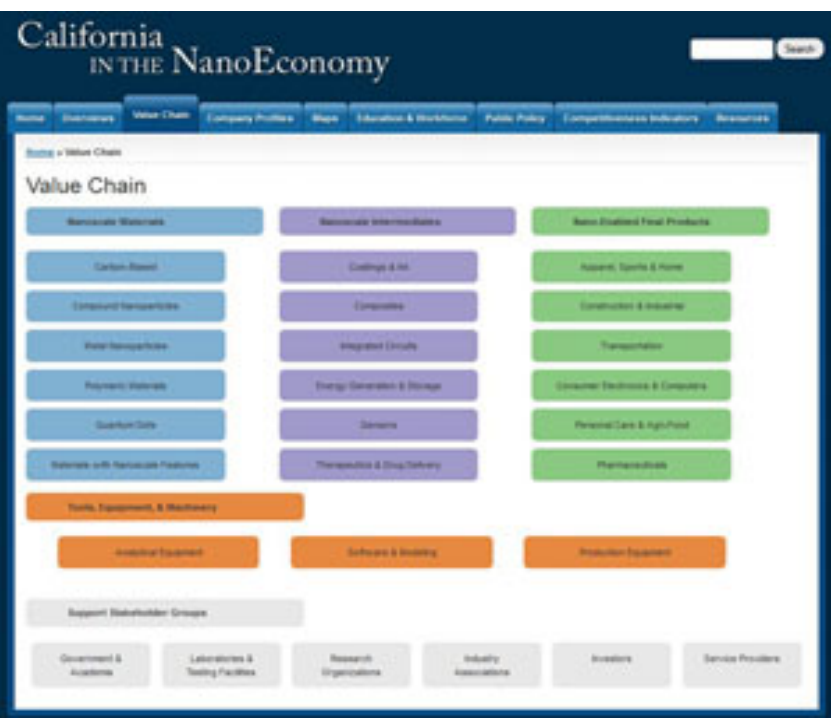

California in the Nano Economy is a new website being launched this September as a resource for the nanotechnology community. The site uses a value chain approach to present California's footprint in nanotechnology including its existing and potential capabilities and resources along the entire chain from nanoscale materials through final products. The site was developed by the Center on Globalization, Governance, \& Competitiveness (CGGC) at Duke University with support from the NSF Center for Nanotechnology in Society (CNS) at the University of California, Santa Barbara.

The purpose of the site is two-fold. The primary focus is to provide a new type of educational resource to enable understanding of the 'nano economy' by using the perspective of the value chain. The approach is illustrated using data for California, and as a result, the site also provides a secondary benefit as a valuable resource for industry in California.

More...

\section{A Small-Scale Solution With a Large-Scale Impact}

\section{Upcoming Calls}

NANOCON 012

Submissions accepted until:

August 31, 2012

SPIE Advanced Lithography Submissions accepted until: September 10, 2012

Call for User Proposals: HighImpact Nanoscience Research Center for Nanophase Materials Sciences Oak Ridge National Laboratory Submissions accepted until: October 17, 2012

View All Calls

Advertisements

STRATEGIES FOR SETTING OCCUPATIONAL EXPOSURE LIMITS FOR ENGINEERED NANOMATERIALS

George Washingston University Washington, DC September 10-11, 2012

\section{Recently Published}

From Our Affiliates

Understanding and correcting for carbon nanotube interferences with a commercial LDH cytotoxicity assay Toxicology 299(2-3)

An analytical model for shearenhanced adhesiveless transfer printing Mechanics Research Communications 43: 46-49

Control of plasmon dynamics in coupled plasmonic hybrid mode microca vities Physical Review 86(4)

Sub-100 nm Si nanowire and nano-sheet array formation by MacEtch using a nonlithographic InAs nanowire mask 


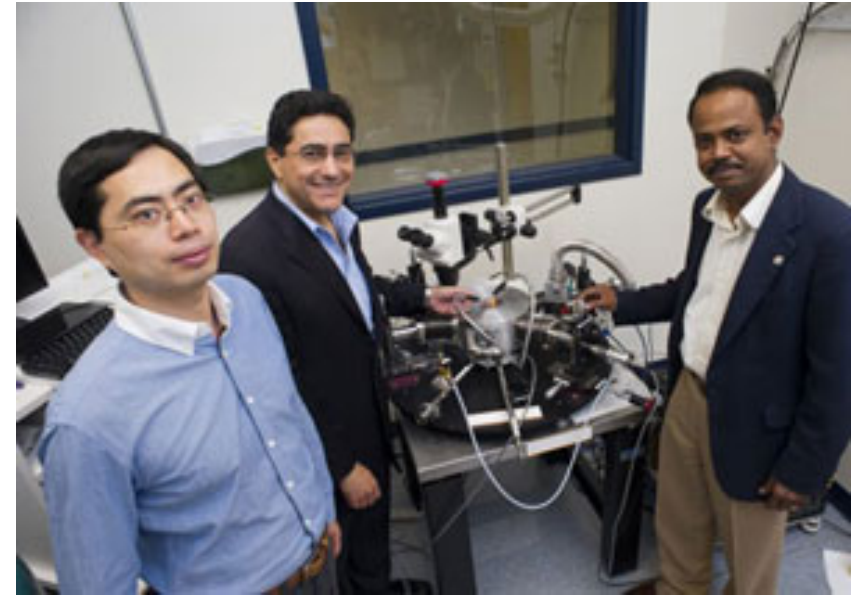

Microchips are pervasive in today's high-tech society, playing integral roles in the inner workings of your cell phone to your Keurig coffee machine.

A processing technology called CMOS, or complementary metal-oxide-semiconductor, made microchips economically feasible in the 1980 s, said Sivasubramanian Somu, a research scientist in Northeastern's Center for High-rate Nanomanufacturing.

A critical element in any microchip is something called an inverter - an electronic component that spits out zeros when you give it ones, and vice versa. "A transistor [the basic element in an inverter] is a simple, extremely fast switch," Somu explained. "You can turn it on and off by electric signals."

More....

\section{NanoBusiness Announces Top 2012 Nanotech Innovators}

Today we announce the Top Nanotech Innovators of 2012. We will be discussing this list in depth at our 11th Annual NanoBusiness / Nanomanufacturing Summit September 4-6th at the Seaport World Trade Center in Boston, MA. This discussion will be part

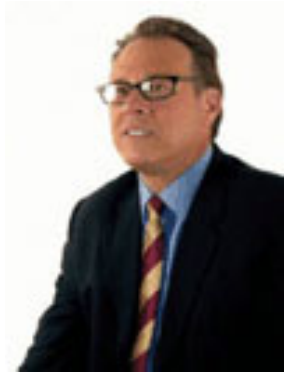
of our program on Thursday, September 6th at $3: P M$. Registration is still open.

This list was created to recognize those companies that are using nanotech to create novel and, in some cases, game-changing technologies. Nanotech is enabling more powerful computer
Nanotechnology 23(30)

Exposure assessment and engineering control strategies for airborne nanoparticles: an application to emissions from nanocomposite compounding processes

Journal of Nanoparticle

Research 14(7)

\section{Affiliated Centers}
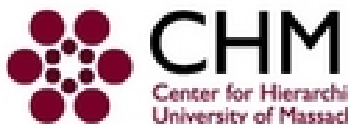

Center for Hierarehical Manufacturing University of Massachusetts Amherst

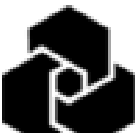

Center for High-rate Nanomanufacturing

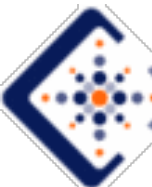

Center for Nanoscale Chemical-Electrical-Mechanical Manufacturing Systems
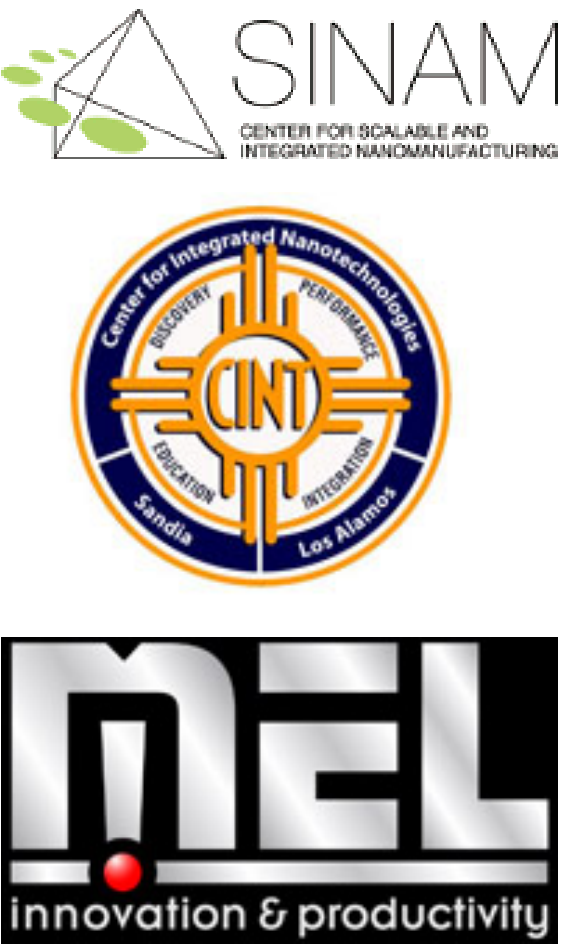
chips, microprocessors, flash memory, and computing devices such as quantum computers, broadband communications systems, solid-state lighting for general illumination, high-performance materials and coatings for transportation, aerospace and various consumer products, synthetic biofuels, nutraceuticals, high-density batteries for transportation and the grid, and targeted-delivery therapeutics for cancer and other life-threatening diseases. As companies continue to innovate at ever-smaller scales, the years ahead promise to be a time of accelerating nanotech commercialization in the global economy.

More....

\section{Read more on InterNano}

Subscribe / Unsubscribe from this list.

Our mailing address is:

The National Nanomanufacturing Network

374 Lederle Graduate Research Center

$710 \mathrm{~N}$. Pleasant Street

University of Massachusetts

Amherst, MA 01003

Our email address is:

nnn@nanomanufacturing.org

Our phone number is:

(413) 577-0570

Copyright (C) 2012 The National Nanomanufacturing Network All rights reserved.

Supported by the National Science Foundation under Grant No. CMMI-1025020. 